\section{Tofacitinib gegen Plaque-Psoriasis auch längerfristig wirksam}

\begin{abstract}
Im vergangenen Jahr wurden die 16-Wochen-Ergebnisse der Psoriasistherapie mit Tofacitinib publiziert. Nun haben die beteiligten Forscher Zwischenergebnisse zur Langzeitanwendung des Wirkstoffs vorgelegt.
\end{abstract}

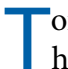
facitinib ist ein oral anwendbarer Inhibitor der Januskinasen 1 und 3. Januskinasen sind Tyrosinkinasen, die an der Weiterleitung extrazellulärer Zytokinsignale in den Zellkern beteiligt sind. Tofacitinib verhindert die Bildung von Entzündungsmediatoren und hat sich in der Therapie der rheumatoiden Arthritis bewährt. In zwei Phase-III-Studien ist der Wirkstoff auch in der Behandlung von insgesamt 1.861 Patienten mit mäßiger bis schwerer chronischer PlaquePsoriasis eingesetzt worden. Nach 16 Wochen waren die Vorteile gegenüber Placebo hochsignifikant ausgefallen.

Eine internationale Forschergruppe hat nun die Egebnisse zur Folgetherapie vorgelegt. Die Probanden der Placebogruppe waren nach 16 Wochen rerandomisiert worden und erhielten dann zweimal täglich Tofacitinib $5 \mathrm{mg}$ oder $10 \mathrm{mg}$. Der Beobachtungszeitraum beträgt mittlerweile bis zu 33 Monate.
\end{abstract}

Die Zwischenresultate nach siebenmonatiger Therapie zeigten unter zweimal täglich $5 \mathrm{mg}$ Tofacitinib bei $55,6 \%$ der Patienten eine mindestens 75\%ige Verbesserung des Psoriasis Area and Severity Index (PASI 75). Unter der 10-mg-Dosierung erreichten $68,8 \%$ einen PASI 75. In der ärztlichen Globalbewertung (Physician's Global Assessment) lautete das Urteil für die 5-mg-Probanden in $54,7 \%$ der Fälle auf „,klares oder nahezu klares Hautbild “. Für die 10-mg-Gruppe betrug dieser Anteil 65,9\%. Das klare Hautergebnis blieb im Wesentlichen über zwei Jahre hinweg erhalten und traf dann noch auf rund $50 \%$ aller Patienten zu. Nach Ablauf des ersten Jahres fand allerdings kein Dosisvergleich mehr statt.

Nasopharyngitiden und Infektionen der oberen Atemwege waren mit 18,8\% und $12,6 \%$ die häufigsten unerwünschten Effekte. Schwere Nebenwirkungen und nebenwirkungsbedingte Therapie- abbrüche betrafen $10,1 \%$ respektive $10,7 \%$ der Probanden. Vier Patienten starben, drei aus der 5-mg-Gruppe (Ursachen: Ösophaguskarzinom, Lungenkrebs, Herzinfarkt) und einer aus der 10-mg-Gruppe (Herzstillstand). Für schwere Infektionen, Herpes zoster und nicht melanomatösen Hautkrebs war eine Abhängigkeit von der TofacitinibDosierung festzustellen. Das Herpeszoster-Risiko nahm mit der Dauer der Exposition zu, knapp 4\% der Probanden erkrankten schließlich daran. Für Malignome als solche, weißen Hautkrebs ausgenommen, war dies nicht der Fall.

Fazit: Die guten 16-Wochen-Ergebnisse für die Psoriasistherapie mit Tofacitinib bestätigen sich auch über einen Zeitraum von zwei Jahren. Schwere Nebenwirkungen treten allerdings häufig auf. Die an den Studien beteiligten Forscher plädieren zusammenfassend dafür, die Substanz weiter auf ihr Potenzial in der Langzeitbehandlung der Psoriasis zu testen.

Dr. Robert Bublak

Papp KA et al. Tofacitinib, an oral Janus kinase inhibitor, for the treatment of chronic plaque psoriasis: Long-term efficacy and safety results from 2 randomized phase-III studies and 1 open-label long-term extension study. J Am Acad Dermatol 2016; 74: 841-50

\title{
Gleichzeitiges Auftreten von primärer und sekundärer Syphilis
}

Ein 68-jähriger Mann hatte seit sechs Wochen eine schmerzlose Erosion an der Innenseite der Oberlippe (Abb. A). An Händen und Füßen zeigten sich zusätzlich schuppende Plaques (Abb. B, C, D), die seit etwa einem Jahr kamen und gingen. In der Labordiagnostik zeigten sich ein positiver TPPA-Assay mit einem Titer von 1:2.560 und ein positiver VDRL-Test mit einem Titer von 1:8. Serologische Untersuchungen auf HIV und Hepatitis verliefen negativ. Der Immunoblot war positiv für Lues-spezifische lgM und IgG.

Die histologische Untersuchung von Biopsien aus der Oberlippenläsion und den Plaques ergab eine Ulzeration und eine psoriasiforme Hyperplasie. Es fanden sich massenhaft CD79A-positive Plasmazellen und Spirochäten.

Unter der Diagnose einer gleichzeitigen primären und sekundären Syphilis wurde der Mann mit Penicillin G Benzathin behandelt. Binnen zwei Monaten kam es zu einer Normalisierung. Ein solches paralleles Auftreten ist ungewöhnlich, aber möglich, da keine Immunität für eine Zweitinfektion besteht. Prof. Hermann S. FüeßI
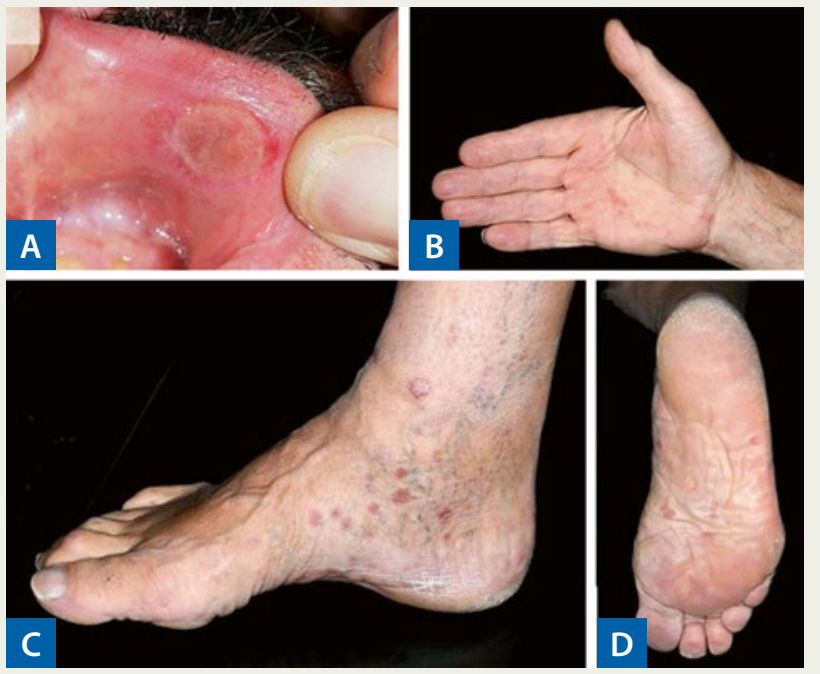

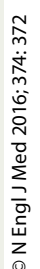

Erosion an der Innenseite der Oberlippe (A), schuppende Plaques an Handinnenflächen (B), Füßen (C) und Fußsohlen (D). 